\title{
Combined nanopore adaptive sequencing and enzyme-based host depletion efficiently enriched microbial sequences and identified missing respiratory pathogens
}

\author{
Mingyu Gan ${ }^{1+}$, Bingbing Wu ${ }^{1+}$, Gangfeng Yan ${ }^{2}$, Gang Li ${ }^{1}$, Li Sun ${ }^{3}$, Guoping $\mathrm{Lu}^{2^{*}}$ and Wenhao Zhou ${ }^{1,4^{*}}$
}

\begin{abstract}
Background: Enzyme-based host depletion significantly improves the sensitivity of clinical metagenomics. Recent studies found that real-time adaptive sequencing of DNA molecules was achieved using a nanopore sequencing machine, which enabled effective enrichment of microbial sequences. However, few studies have compared the enzyme-based host depletion and nanopore adaptive sequencing for microbial enrichment efficiency.

Results: To compare the host depletion and microbial enrichment efficiency of enzyme-based and adaptive sequencing methods, the present study collected clinical samples from eight children with respiratory tract infections. The same respiratory samples were subjected to standard methods, adaptive sequencing methods, enzyme-based host depletion methods, and the combination of adaptive sequencing and enzyme-based host depletion methods. We compared the host depletion efficiency, microbial enrichment efficiency, and pathogenic microorganisms detected between the four methods. We found that adaptive sequencing, enzyme-based host depletion and the combined methods significantly enriched the microbial sequences and significantly increased the diversity of microorganisms ( $p$ value $<0.001$ for each method compared to standard). The highest microbial enrichment efficiency was achieved using the combined method. Compared to the standard method, the combined method increased the microbial reads by a median of 113.41-fold (interquartile range 23.32-327.72, maximum 1812), and the number of genera by a median of 70 -fold (interquartile range $56.75-86.75$, maximum 164). The combined method detected 6 pathogens in 4 samples with a median read of 547 , compared to 5 pathogens in 4 samples with a median read of 4 using the standard method.
\end{abstract}

\footnotetext{
* Correspondence: 13788904150@163.com; zhouwenhao@fudan.edu.cn

${ }^{\dagger}$ Mingyu Gan and Bingbing Wu contributed equally to this work.

${ }^{2}$ Department of Pediatric Emergency and Critical Care Medicine, Children's

Hospital of Fudan University, National Children's Medical Center, 399

Wanyuan Road, Shanghai 201102, China

${ }^{1}$ Center for Molecular Medicine, Pediatric Research Institute, Children's

Hospital of Fudan University, National Children's Medical Center, Shanghai,

China

Full list of author information is available at the end of the article
}

C C The Author(s). 2021 Open Access This article is licensed under a Creative Commons Attribution 4.0 International License, which permits use, sharing, adaptation, distribution and reproduction in any medium or format, as long as you give appropriate credit to the original author(s) and the source, provide a link to the Creative Commons licence, and indicate if changes were made. The images or other third party material in this article are included in the article's Creative Commons licence, unless indicated otherwise in a credit line to the material. If material is not included in the article's Creative Commons licence and your intended use is not permitted by statutory regulation or exceeds the permitted use, you will need to obtain permission directly from the copyright holder. To view a copy of this licence, visit http://creativecommons.org/licenses/by/4.0/ The Creative Commons Public Domain Dedication waiver (http://creativecommons.org/publicdomain/zero/1.0/) applies to the data made available in this article, unless otherwise stated in a credit line to the data. 
Conclusion: The combined method is an effective, easy-to-run method for enriching microbial sequences in clinical metagenomics from sputum and bronchoalveolar lavage fluid samples and may improve the sensitivity of clinical metagenomics for other host-derived clinical samples.

Keywords: Metagenomics, Nanopore adaptive sequencing, Host depletion, Microbe enrichment

\section{Background}

Infectious diseases remain the major threat to human health, especially for children under the age of 5 [1]. Rapid and accurate detection of the causative agent is the key to treatment [2]. With the development of sequencing technology, metagenomics sequencing has been widely used for clinical pathogen detection [3-5]. Metagenomic sequencing is able to sequence all nucleic acids in samples and detect all potential pathogens, even emerging pathogens [6-8].

However, a high background of host DNA in clinical samples impedes the detection of pathogens [9]. This shortage of clinical metagenomics may be overcome via microbe enrichment [3,9-11]. 16S rDNA sequencing is very effective in profiling microbial diversity [12-14]. However, 16S sequencing for clinical diagnosis misses important viral and fungal pathogens in the respiratory tract. The direct enrichment of viruses using spiked primers achieved a median of tenfold enrichment [15]. However, this type of method only enriches limited number of microbes, which nullifies the major advantage of metagenomic sequencing. Experimental host DNA depletion methods enable relative microbe enrichment $[3,16,17]$. Charalampous et al. used the saponin-based differential lysis method to deplete host DNA, which resulted in a maximum $10^{4}$-fold depletion of host DNA and maximum 100-fold enrichment of microbe DNA [3].

Nanopore sequencing is characterized by long read sequencing and real-time data analysis, which is important for the rapid identification of pathogens and suspected emerging pathogens $[3,18,19]$. Nanopore sequencing allows computationally targeted sequencing, which is known as Read Until mode [20]. Signals in this mode were analyzed in real-time after a DNA molecule entered a pore. The beginning of the sequence strand was rapidly mapped to the provided reference sequence. If the sequence was located in the targeted region, or was not a sequence to be depleted, the DNA molecule was allowed to continue sequencing. If the sequence was not the targeted sequence, or was to be depleted, the DNA molecule was ejected from the pore. Based on this function, Alexander et al. developed a toolkit (readfish) and showed a 5.7 -fold increase in a relatively low abundance microbe in the ZymoBIOMICS mock metagenomic community [21]. Another study developed UNCALLED software, which uses raw signals to compare with the reference sequence. They showed a 4.46 -fold enrichment of yeast sequence [22].

However, few studies applied nanopore adaptive sequencing to clinical samples for microbe enrichment. The difference in enrichment efficiency between enzyme-based host depletion and nanopore adaptive sequencing is not known. Alternatively, the best approach may be to combine these two methods to efficiently enrich microbes. The present study hypothesized that the combination of experimental host depletion and adaptive sequencing would produce the best microbe enrichment efficiency compared to the use of either method alone. Four groups of results for each clinical sample were acquired by combining standard DNA extraction and enzyme-based host depletion with standard and adaptive sequencing. We showed a median of 113.41-fold microbe enrichment using the combination method of enzyme-based host depletion and adaptive sequencing.

\section{Results}

\section{Study design}

To evaluate the microbe enrichment efficiency of experimental enzyme-based host depletion, nanopore adaptive sequencing and the combination of the two methods, we designed the experimental procedure illustrated in Fig. S1. At first, each sample was divided into two parts with the same volume. Each part was processed using standard DNA extraction and enzyme-based host DNA depletion respectively. For each DNA, standard sequencing and adaptive sequencing were performed simultaneously on the same flow cell. Therefore, each sample was processed using four methods: standard DNA extraction with standard sequencing (SD_SSD, the standard), standard DNA extraction with adaptive sequencing (SD_ADS, using adaptive sequencing alone), host depletion with standard sequencing (HD_SSD, using enzyme-base host depletion alone), and host depletion with adaptive sequencing (HD_ADS, the combined method). We collected 8 sputum or bronchoalveolar lavage fluid (BALF) samples from children with pneumonia or pulmonary infection (Table S1).

\section{Nanopore sequencing results}

Nanopore sequencing was performed on the ONT GridION platform. Because samples were sequenced on different flow cells with various numbers of active pores, 
the reads and bases generated were not equal between samples (Table S2). For each sample, adaptive sequencing and standard sequencing were performed on the same flow cell by setting the adaptive sequencing channel from 1 to 256. Therefore, we compared the sequencing outputs between adaptive sequencing and standard sequencing. There was no consistent trend between the number of reads yielded by adaptive and standard sequencing. However, except for the enzyme host depleted sample of P1, adaptive sequencing yielded fewer bases than standard sequencing in all of the other samples. This result may be caused by the shorter sequence length of adaptive sequencing (Table S2 and Fig. S2).

\section{Adaptive sequencing reads}

The Oxford nanopore sequencer enables selective sequencing by controlling the driving voltage across individual nanopores. Therefore, three types of reads are yielded from adaptive sequencing. According to Oxford nanopore's definition, "unblock" reads represent the DNA molecules rejected by the pore, and the first few hundred bp of these DNA molecules are sequenced. The "stop receiving" reads mean DNA molecules accepted by the pore, and the full length of the molecules should be sequenced. The "no decision" reads indicate that DNA molecules cannot be decide to be rejected or accepted. We compared the percentage of the three types of reads. We observed "stop receiving" reads in the SD_ADS group (Fig. S3A), but few in the HD_ADS group (Fig. $\mathrm{S} 3 \mathrm{~B})$. The percentage of "stop receiving" reads in the SD_ADS group was lower than the "unblock" and "no decision" reads in each sample. However, the long length of "stop receiving" reads resulted in an increased percentage of bases (Fig. S3A, Fig. S4A). For the "unblock" and "no decision" reads, we found that except for the P4 and P6 samples, the "unblock" reads and bases were higher than the "no decision" reads and bases in the HD_ADS group (Fig. S3B). However, we didn't observe the same trend in SD_ADS (Fig. S3A). The length of the "unblock" reads was smaller than the "no decision" reads in both groups, and the length of the "unblock" reads was smaller than the "stop receiving" reads (Fig. S4). The "stop receiving" reads indicate the accepted reads, which means the microbial reads in our study. However, we found that on average $93.42 \%$ of the "stop receiving" reads could be mapped to the human genome.

\section{Human sequence depletion}

The enzyme-based host depletion (HD) method resulted in shorter reads than standard DNA extracted (SD) method (Fig. S2). Most of the long reads of the SD group were classified as human (e.g. greater than $99 \%$ of the reads longer than $5000 \mathrm{bp}$ belonged to humans), which indicates that long human reads were depleted by the HD method. Adaptive sequencing (ADS) also significantly depleted long human reads (Fig. S2).

Reads were classified into four classes, including human, E. coli, unclassified and microbe reads (Table S3). We observed that a large part of the nonhuman reads in the SD_SSD and SD_ADS groups were classified as $E$. coli (Table S3). However, most of these reads only mapped to a specific region in the E. coli genome (Fig. S5), which suggests reagent contamination in the Ligation Sequencing Kit (SQK-LSK109). After excluding unclassified and E. coli reads, the relative abundances of human and microbe reads in each sequencing sample were calculated. A significant decrease in human reads was found in four samples (P4, P6, P7 and P8) but only between the SD and HD groups (Fig. 1, Table S3). For human bases, a continuous significant decrease in human bases was found in four samples (P4, P6, P7 and P8), following the order of SD_SSD, SD_ADS, HD_SSD and HD_ADS (Fig. 1, Table S3). The reduction in bases indicates that in addition to the HD method, the ADS method was also effective in removing human sequences. The different reduction patterns between human reads and bases occurred because the ADS method requires the identification of a short sequence to determine whether it is human. This step results in the output of short human reads (i.e., the "unblock" reads illustrated in Fig. S3 and Fig. S4).

\section{Microbial sequence enrichment}

The respiratory tract contains a wide variety of colonizing microorganisms. After identifying the microbial species of the sequenced reads, we summed the reads of all microorganisms. We compared the normalized number of reads and bases of microorganisms obtained using the four methods. We found that the HD and ADS methods alone and the combined HD and ADS methods were significantly enriched for microorganisms (Fig. 2, Table S4). The results of all four methods were significantly different from each other $(p$ value $<0.001)$. The HD method alone enriched more microorganisms than the ADS method alone. The combination of the HD and ADS methods enriched most microorganisms. Compared to the standard method, ADS alone enriched microbial reads by a median of 3.59-fold (interquartile range $2.39-10.34$, maximum 33 ), $\mathrm{HD}$ alone by a median of 62.96-fold (interquartile range 18.18-144.12, maximum 1072), and the combined method HD_ADS by a median of 113.41-fold (interquartile range 23.32-327.72, maximum 1812).

\section{Increased microbial diversity}

We also compared the number of genera and alpha diversity of the microorganisms detected using the four methods (Fig. 3). We found that, similar to the microbial 

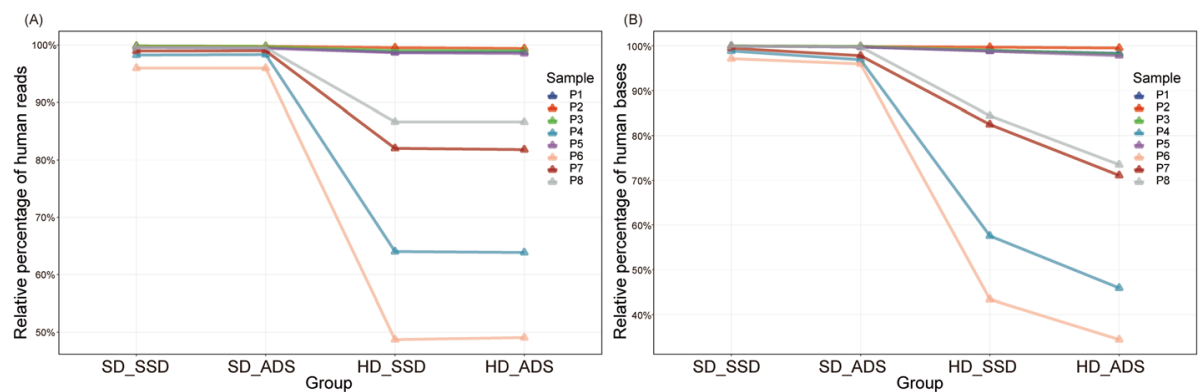

Fig. 1 Human sequence depletion. Relative abundance of (A) host reads, and (B) host bases

sequence enrichment results, the HD and ADS methods alone and the combined method significantly increased the number of genera and alpha diversity. The number of microbial genera and alpha diversity increased more with the HD method alone than with the ADS method alone. The combined method had the highest enrichment efficiency for microbial genera (median 70-fold, interquartile range 56.75-86.75, maximum 164). However, there was no significant difference in alpha diversity between the combined method and the HD method alone, ADS method alone and HD method alone.

The top 15 microorganisms in each sample are shown in Fig. 4. Except the increase microbial diversity, we also found that the HD, ADS and the combined methods increased the sequencing reads for each microorganism (Fig. 4). For these top 15 microorganisms, ADS method increases the reads by a median of 2.83-fold (interquartile range 2.34-4.16, maximum 19), HD method increases the reads by a median of 37.17-fold (interquartile range 15.31-163.15, maximum 1232), the combined method increases the reads by a median of 47.14-fold (interquartile range 21.56-278.75, maximum 2081).

\section{Pathogenic microorganisms detected}

The aim of enriching microorganisms by host depletion is to increase the detection sensitivity of pathogenic microorganisms. Therefore, we compared the pathogenic microorganisms detected using the four methods. We invited experienced laboratory experts to identify pathogenic microorganisms from microbial classification results by reviewing medical records. Possible pathogens were identified in 4 patients (Table 1). For the other 4 patients, we did not detect any pathogenic microorganisms in the standardized results (Table S4) or the raw results (Table S5). Patient P4's chest X-ray suggested pneumonia and severe lung infection. S. pneumoniae was detected by all four methods, and the number of reads increased progressively in the order of SD_SSD, SD_ADS, HD_SSD and HD_ADS. The child had a pulmonary infection and was given cefotaxime. However, the child suffered from acute liver failure and was in critical condition. The family requested to abandon the treatment and the child was discharged automatically. Patient P6 had recurrent fever, cough, shortness of breath, coarse breath sounds in both lungs, and pulmonary exudate as suggested by CT. S. pneumoniae was
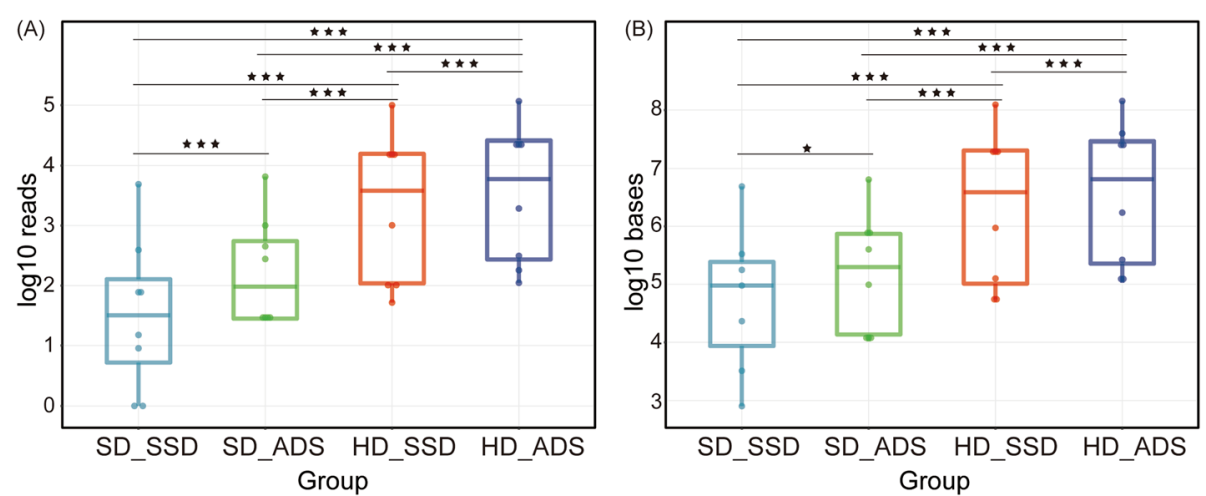

Fig. 2 Microbial sequence enrichment. A Microbial reads and (B) microbial bases detected by the four methods. SD_SSD: the standard method, SD_ADS: using adaptive sequencing alone, HD_SSD: using enzyme-base host depletion alone, HD_ADS: the combined method. Significant difference was found between each combination among the four methods 

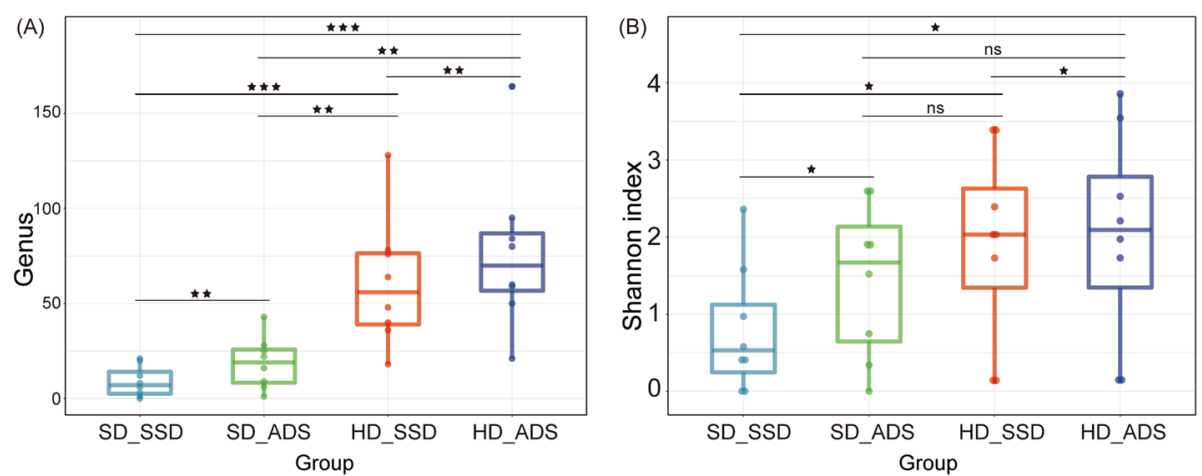

Fig. 3 Microbial diversity. A Genus detected by the four method. B Alpha diversity of the four methods

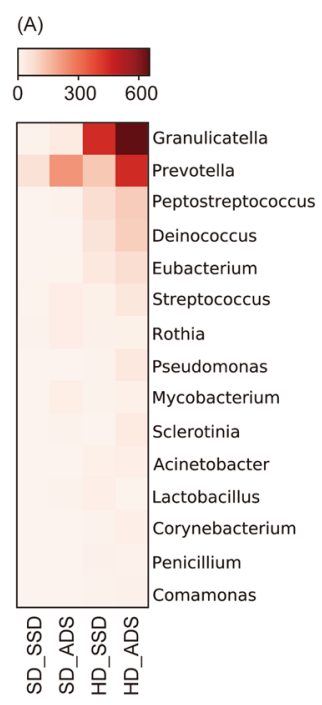

(E)
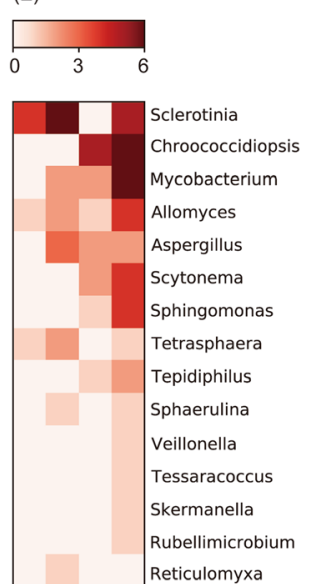

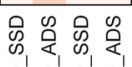

응 올 오
(B)

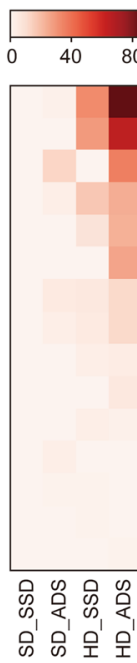

(F)

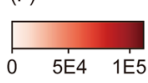

Streptococcus

Lactobacillus Rothia

Eubacterium

Selenomonas

Mycobacterium

Pseudomonas

Granulicatella

Enterococcus

Tetrasphaera

Acinetobacter

Sclerotinia

Corynebacterium Staphylococcus Prevotella

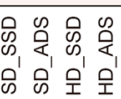

(C)

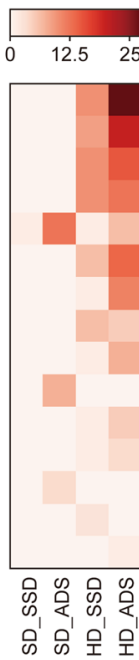

(G)

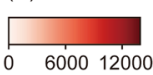

Streptococcus Rothia Gemella Granulicatella Actinomyces Haemophilus Atopobium Parvimonas Prevotella Neisseria Abiotrophia Leptotrichia Solobacterium Fusobacterium Corynebacterium

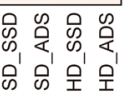

(D)

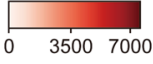

Prevotella Atopobium Streptococcus Veillonella Trueperella Rothia Actinomyces Granulicatella Alloprevotella Pseudomonas Porphyromonas Lactobacillus Mycobacterium Psychromonas

总总总总 Bacteroides

(H)

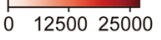

Tropheryma Acinetobacter Pseudomonas Sphingomonas Prevotella Rhodococcus Burkholderia Rhizobium Methylobacterium Meyerozyma Sphingobium Gordonia Penicillium Sphingopyxis Xenophilus

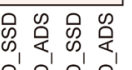

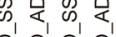

的的呈重

Fig. 4 Top 15 microbial genus detected by the four methods in each sample. A-H indicates P1-P8 patients 


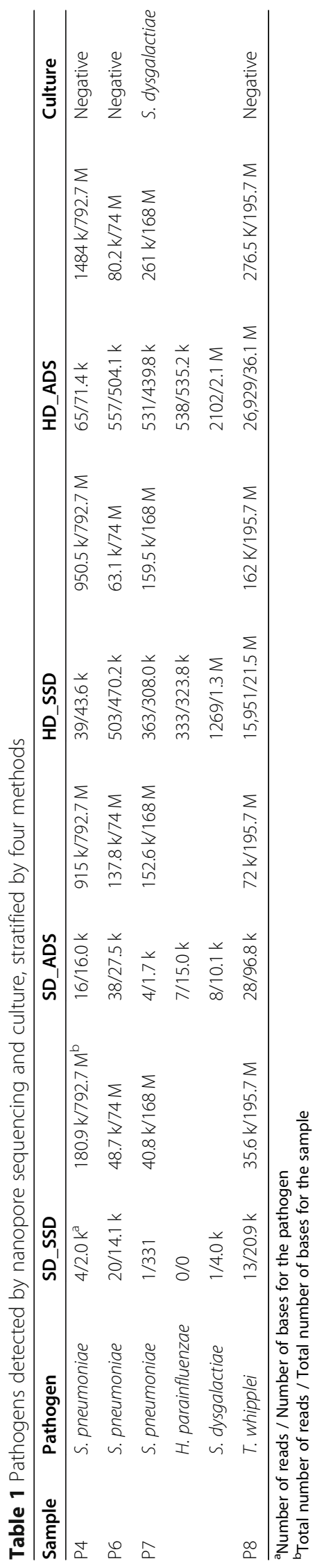


detected by all four methods, and the number of reads increased progressively in the order of SD_SSD, SD_ADS, HD_SSD and HD_ADS. The child was diagnosed with severe combined immunodeficiency disease (IL2RG gene mutation) and was given meropenem, fosfomycin, voriconazole, caspofungin, teicoplanin, and sulfofen for antiinfection. However, the child was heavily infected and had multiple organ insufficiency. After aggressive antiinfective treatment, the child still had a fluctuating temperature, and no signs of improvement of multiple organ insufficiency. After careful consideration, the child's family requested a discharge. Patient P7 had juvenile idiopathic arthritis, and a CT scan of the chest showed multiple ground glass shadows in both lungs. BALF was collected for further diagnosis of connective tissue disease-associated interstitial lung disease and lung infection. S. pneumoniae and S. dysgalactiae were detected by all four methods. $H$. parainfluenzae was detected by three methods except SD_SSD. S. dysgalactiae was also detected by culture. According to the culture results, anti-infection treatment with ampicillin and sulbactam was administered intravenously. The child was cured and discharged from the hospital. Patient P8 had childhood dermatomyositis and was admitted with a diagnosis of juvenile dermatomyositis involving the lungs. Lung CT showed localised interstitial changes in both lungs. With the concern of a specific pathogenic opportunistic infection, BALF was collected. Consistent with clinician's concern, an extremely rare pathogen was detected. T. whipplei was detected by all four methods, and the number of reads detected had the same trend as the rest of the patients. The child was treated with ceftriaxone for 2 weeks in combination with oral anti-infective treatment with sulfamethoxazole. After treatment, the child was in good general condition and was discharged.

\section{Discussion}

The extremely high proportion of host nucleic acids in clinical samples can drown out microbial sequences and has important implications for the sensitivity of clinical metagenomics $[9,10]$. The present study compared the microbe enrichment efficiency of enzyme-based host depletion, adaptive sequencing, and the combination of these two methods. We found that all three methods significantly enriched the sequences of microorganisms. The sequencing read enrichment efficiency of the combined method was significantly higher than the other methods and reached a median of 113.41-fold. The three methods also significantly increased the diversity of microorganisms detected. The combined method had the highest enrichment efficiency for microbial genera (median 70-fold), which was significantly higher than the other methods. The results for pathogens detected followed the same trend, with the combined method achieving the highest positivity rate, the number of pathogenic microorganisms detected and the corresponding number of reads. The results of this study provide a new microbial enrichment strategy for clinical metagenomics.

Enrichment of microorganisms effectively increases the positive rate of clinical metagenomics [10, 15, 23]. Current enrichment methods are divided into two major groups: methods that directly enrich microorganisms and methods that deplete the hosts. 16S sequencing directly enriches the 16S rRNA of microorganisms and is widely used to study microbial communities in various human ecological niches. These niches include microbial-rich sites (i.e., the gastrointestinal tract, the lower respiratory tract) and microbial-rare sites (i.e., blood and womb, which are normally considered sterile) [12-14]. It demonstrates the strong enrichment ability of $16 \mathrm{~S}$ sequencing. However, for clinical diagnosis, $16 \mathrm{~S}$ sequencing is not as applicable as metagenomics sequencing because it cannot detect viruses and fungi. In addition, short-sequencing of $16 \mathrm{~S}$ sub-regions does not fully satisfy microbial specielevel identification [24]. The spiked-primer-based method targets 14 viruses, and achieves a median of 10 -fold enrichment [15]. However, the targets for microbial enrichment are generally limited, which loses the major advantage (untargeted detection) of metagenomics $[15,25$, 26]. Host depletion methods include filter-based, CpG methylation-based, mammalian cell selective lysis, and propidium monoazide-based methods [11, 16]. Filterbased methods cannot deplete extracellular microbial DNA [16]. Methylation-based methods are not suitable for microorganisms with methylation patterns that are similar to eukaryotes. Selective lysis depletes host DNA by first lysing the host cell then degrading the released host DNA. Selective lysis is the most widely used method in clinical metagenomics. Real-time nanopore adaptive sequencing recently enabled the efficient enrichment of target sequences [21, 22]. This method compares sequencing reads to target sequences in real time, which enable realtime control of DNA molecules. Because the method achieves targeted enrichment during sequencing, the principle is completely different from the previous method, which makes it possible to use the two methods in combination. The results of this study also demonstrated that combining the two methods was more effective than using either method alone.

We observed a significant reduction in the relative proportions of host reads and bases in four samples (P4, P6, P7 and P8), but the remaining four samples showed no significant change. This result may be associated with the absolute content of microbial sequences in the samples. We observed that in the standard group without any methodological treatment (SD_SSD), significantly higher microbial sequences were detected in P4, P6, P7 
and P8 than the other 4 samples, and the microbial content also correlated with the trend of host depletion. This result suggests that higher microbial content improves the host depletion effect for the clinical samples.

The present study used GRCh38 as the reference sequence to remove human sequences. However, the inclusion of partially inserted viral sequences in this reference genome may have resulted in poor enrichment efficiency for this class of viruses [27]. Although no sequences derived from this class of viruses were observed in this study, future studies should modify the reference sequence to obtain the corresponding viral sequence.

Two patients enrolled in this study had a clinical diagnosis of interstitial pneumonia with suspected viral infection. However, we did not detect the virus using any of the four methods. This result may be because that the viral capsid is more fragile than the cell wall of bacteria, which resulted in the degradation of viral DNA during selective lysis. Previous studies also suggested that selective lysis led to a bias towards Gram-positive bacteria [28]. Therefore, the limitations of this method must be considered when using a selective lysis method.

One limitation of this study is the limited sample size, which made it impossible to compare the positive detection rates of pathogenic microorganisms between the different methods. However, our results showed that the combined method significantly enriched the microorganisms. Another limitation is the lack of validation of the pathogenic microorganisms detected. The positive rate of culture was too low to validate the results of the metagenomics testing. However, with the exception of patient P8, the pathogenic microorganisms identified were all common respiratory pathogens, including hospital-acquired pathogenic microorganisms. Patient P8 has childhood dermatomyositis. He was treated with long-term oral high-dose hormone and immunosuppressive therapy, which may lead to opportunistic infections with specific pathogens. Because of the extremely low concentration of DNA after host depletion, the starting conditions for ligation sequencing library construction (SQK-LSK109) were not met. Therefore, we used a PCR-based library construction kit (SQK-PSK004). This resulted in different library construction procedure for the HD and SD samples. The effect of PCR on the results during the library constructing process cannot be excluded.

\section{Conclusions}

The present study showed that the combination of enzyme-based host depletion and nanopore adaptive sequencing reached the highest microbial enrichment efficiency and positive detection rate of pathogenic microorganisms. This study provides a new strategy for microbial enrichment and improvement of the positive detection rate of pathogenic microorganisms.

\section{Methods}

\section{Clinical sample collection}

Patients in the Children's Hospital of Fudan University with an admission diagnosis of pneumonia or pulmonary infection were prospectively enrolled (Table S1). BALF or sputum samples were collected from these patients.

\section{Microbe culture}

BALF samples were sent to the Department of Clinical Laboratory for bacteria culture. Culture and strain identification were performed using a VITEK2 COMPACT automated ID/AST instrument (bioMérieux, France), as per the manufacturer's instructions.

\section{DNA extraction and enzyme-based host depletion}

BALF or sputum samples were divided into two parts (200 ul each) for standard DNA extraction and host DNA depletion. Standard DNA extraction was performed using the MagMAX CORE Nucleic Acid Purification kit per the manufacturer's instructions.

Two types of methods exist for the enrichment of microorganisms in clinical samples. The first method is the direct capture of microbial sequences using primers or probes, but the target of these types of methods is limited. The second type is the reverse enrichment of microorganisms by removing the host nucleic acid. For example, a 5-um filter was used to remove intact human cells. The NEBNext kit enriches microorganisms by selective binding and removal of CpG-methylated host DNA. Microorganisms may also be enriched by the differential lysis of the host cells and removal of the released DNA. The differential lysis method was used to enrich pathogenic microorganisms in this study. Host DNA depletion and microbial DNA isolation were performed using a HostZERO Microbial DNA kit per the manufacturer's instructions.

\section{Nanopore library preparation and sequencing}

Approximately $1 \mu \mathrm{g}$ of DNA extracted by the standard method was used to prepare a sequencing library using the Ligation Sequencing Kit (SQK-LSK109) according to the manufacturer's instructions. For library cleanup, short fragment buffer (SFB) was used to retain DNA fragments of all sizes. The sequencing library for DNA extracted by the HostZERO Microbial DNA kit was prepared using the PCR Sequencing Kit (SQK-PSK004), according to the manufacturer's instructions. Approximately $50 \mathrm{fmol}$ of the prepared library was loaded onto the R9.4.1 flow cell. Sequencing was performed using the ONT GridION sequencing platform. Adaptive sequencing was applied using MinKNOW (21.10.6) software, which allowed us to deplete human sequences dynamically. Adaptive sequencing and standard sequencing for each library were performed simultaneously on the same flow cell by setting 
the adaptive sampling channel from 1 to 256 (leave 257512 channels for standard sequencing). GRCh38 was used as the reference sequence. Host sequence depletion was enabled by selecting "Deplete".

\section{Nanopore sequence analysis}

The Fastq file was processed with porechop v0.2.4 (https:/github.com/rrwick/Porechop) with the default parameters to trim off sequencing adapters. Trimmed reads were aligned to GRCh38 using minimap2 (2.17) with the parameters "- $x$ map-ont -a -t 8" [29]. The preset option "-x map-ont" applies 15 as the minimizer kmer length. The other minimap2 parameters were all set as default. As long as the read mapped to the GRCh38 reference, it was considered to be human, even when the value of mapping quality was 0 , i.e., multiple mapped read. Human reads were set aside. The remaining unmapped reads were considered nonhuman reads. They were extracted from the sam format file using samtools with the command "samtools view -f 4" [30].

The nonhuman reads were aligned with our microorganism reference database using centrifuge (1.0.3) [31] with default parameters. The microorganism reference database was built by downloading Refseq-level sequences from the NCBI genome database (ftp://ftp.ncbi.nlm.nih.gov/ genomes/). Sequences shorter than $150 \mathrm{bp}$ were deleted. The final database was comprised of more than 20,000 reference genomes, including over 12,000 bacteria, 7312 viruses, 515 fungi, and 168 parasites. Reads aligned to multiple species were filtered out. Reads less than $100 \mathrm{bp}$ in length were filtered out. Reads with sequences less than 50 bp aligned to the reference genome were also filtered out.

\section{Microbe identification}

We developed a non-template-control (NTC)-based strategy to differentiate microbes from the experiment and reagent contamination. NTC was added for each batch of experiments, from DNA extraction to sequencing. After microorganism reference alignment and read filtering, standardized bases (bases per million sequencing bases (BPM), calculated as bases of a specific microbe/total sequencing bases $\times 1,000,000$ ) were calculated for the sample and NTC. If a microbe was detected in the NTC, a BPM ratio (calculated as BPM-Sample/BPM-NTC) of 5 was used as the cut-off to differentiate the true positive microbes from the background. If a microbe was not detected in the NTC, a BPM value of 1 was used for reporting.

\section{Fastq normalization}

Because the samples were sequenced on different flow cells, the number of reads obtained for each sample was not equal. To compare the removal of human reads and the microbial enrichment efficiency of the same sample by the four methods, we normalized the fastq data. Due to the uneven length of the sequenced reads, we normalized the four types of sequencing data for each sample according to the number of bases sequenced. Sequencing reads were randomly selected from each fastq file, until the total bases reach the standard number. The four fastq files sequenced from the same sample were processed with the same total base number.

\section{Statistical methods}

We used a paired t-test in $\mathrm{R}$ (v4.1.0) to calculate the $p$ value for the comparison of microbial reads, bases, number of genera and alpha diversity between the four methods. The number of microbial reads and bases were $\log 10$ transformed for $\mathrm{p}$ value statistics.

\section{Abbreviations}

SD_SSD: Standard DNA extraction with standard sequencing;

SD_ADS: Standard DNA extraction with adaptive sequencing; HD_SSD: Host depletion with standard sequencing; HD_ADS: Host depletion with adaptive sequencing; BALF: Bronchoalveolar lavage fluid; HD: Host depletion;

SD: Standard DNA extraction; ADS: Adaptive sequencing; NTC: Non-templatecontrol; BPM: Bases per million sequencing bases

\section{Supplementary Information}

The online version contains supplementary material available at https://doi. org/10.1186/s12864-021-08023-0.

Additional file 1: Fig S1. Study design.

Additional file 2: Fig S2. Nanopore sequencing read length of the four group, illustrated using P7 sample.

Additional file 3: Fig S3. Relative proportion of sequencing reads and bases of adaptive sequencing output. (A) SD_ADS group, (B) HD_ADS group.

Additional file 4: Fig S4. Read length of adaptive sequencing, illustrated using P4 sample. (A) SD_ADS group, (B) HD_ADS group. "unblock": rejected reads, "stop receiving": accepted reads, "no decision": reads without decision.

Additional file 5: Fig S5. Mapping coverage of E.coli reads, illustrated using P1 sample processed with the SD_ADS method.

Additional file 6: Table S1. Patients enrolled in this study.

Additional file 7: Table S2. The number of reads and bases yielded by nanopore sequencing.

Additional file 8: Table S3. Relative proportion of human, E.coli, unclassified and microbe reads and bases in each sample.

Additional file 9: Table S4. Taxonomy classification results of the top 50 microorganisms in each sample obtained using normalized fastq files. For samples with the number of microbial species less than 50, all their microbial classification results were shown.

Additional file 10: Table S5. Taxonomy classification results of the top 50 microorganisms in each sample obtained using raw fastq files. For samples with the number of microbial species less than 50, all their microbial classification results were shown.

\section{Acknowledgements}

Not applicable.

Authors' contributions

WZ and GL1 conceived the project. MG performed computational analyses. MG performed the experiments with the help of GL2. GY and LS had roles in recruitment, data collection and data interpretation. MG and BW wrote the 
manuscript. All authors have read and approved the manuscript. GL1: Guoping Lu. GL2: Gang Li.

\section{Funding}

This work was supported by Science and Technology Commission of Shanghai Municipality (19495810300) and Shanghai Hospital Development Center (SHDC22020217). The funding body played no role in the design of the study and collection, analysis, and interpretation of data and in writing the manuscript.

\section{Availability of data and materials}

The raw nanopore sequencing reads, after excluding human reads, have been deposited into BioProject (accession number PRJNA763343). The reference human genome used in this study was downloaded from NCBI (https://www.ncbi.nlm.nih.gov/assembly/GCF_000001405.26/)

\section{Declarations}

\section{Consent to publication}

Not applicable.

\section{Ethics approval and consent to participate}

This study was approved by the ethics committees of Children's Hospital of Fudan University (2019-300) and written informed consent for participation in the study was obtained where participants are children (under 16 years old) from their parent or guardian. This study was performed in line with the principles of the Declaration of Helsinki.

\section{Competing interests}

The authors have no competing interest to declare that are relevant to the content of this article.

\section{Author details}

'Center for Molecular Medicine, Pediatric Research Institute, Children's Hospital of Fudan University, National Children's Medical Center, Shanghai, China. ${ }^{2}$ Department of Pediatric Emergency and Critical Care Medicine, Children's Hospital of Fudan University, National Children's Medical Center, 399 Wanyuan Road, Shanghai 201102, China. ${ }^{3}$ Department of Rheumatology, Children's Hospital of Fudan University, National Children's Medical Center, Shanghai, China. ${ }^{4}$ Department of Neonates, Key Laboratory of Neonatal Diseases, Ministry of Health, Children's Hospital of Fudan University, National Children's Medical Center, Shanghai, China.

\section{Received: 28 May 2021 Accepted: 17 September 2021}

\section{Published online: 09 October 2021}

\section{References}

1. Liu L, Oza S, Hogan D, Chu Y, Perin J, Zhu J, et al. Global, regional, and national causes of under-5 mortality in 2000-15: an updated systematic analysis with implications for the sustainable development goals. Lancet. 2016:388(10063):3027-35. https://doi.org/10.1016/S0140-6736(16)31593-8.

2. Barlam TF, Cosgrove SE, Abbo LM, MacDougall C, Schuetz AN, Septimus EJ, et al. Implementing an antibiotic stewardship program: guidelines by the Infectious Diseases Society of America and the Society for Healthcare Epidemiology of America. Clin Infect Dis. 2016;62(10):e51-77. https://doi. org/10.1093/cid/ciw118.

3. Charalampous T, Kay GL, Richardson H, Aydin A, Baldan R, Jeanes C, et al. Nanopore metagenomics enables rapid clinical diagnosis of bacterial lower respiratory infection. Nat Biotechnol. 2019;37(7):783-92. https://doi.org/10.1 038/s41587-019-0156-5.

4. Blauwkamp TA, Thair S, Rosen MJ, Blair L, Lindner MS, Vilfan ID, et al. Analytical and clinical validation of a microbial cell-free DNA sequencing test for infectious disease. Nat Microbiol. 2019;4(4):663-74. https://doi.org/1 0.1038/s41564-018-0349-6.

5. Wilson MR, Sample HA, Zorn KC, Arevalo S, Yu G, Neuhaus J, et al. Clinical metagenomic sequencing for diagnosis of meningitis and encephalitis. $\mathrm{N}$ Engl J Med. 2019;380(24):2327-40. https://doi.org/10.1056/NEJMoa1803396.

6. Wu F, Zhao S, Yu B, Chen YM, Wang W, Song ZG, et al. A new coronavirus associated with human respiratory disease in China. Nature. 2020:579(7798): 265-9. https://doi.org/10.1038/s41586-020-2008-3.
7. Wilson MR, Naccache SN, Samayoa E, Biagtan M, Bashir H, Yu G, et al. Actionable diagnosis of neuroleptospirosis by next-generation sequencing. N Engl J Med. 2014;370(25):2408-17. https://doi.org/10.1056/NEJMoa14012 68.

8. Ai JW, Weng SS, Cheng Q, Cui P, Li YJ, Wu HL, et al. Human Endophthalmitis caused by pseudorabies virus infection, China, 2017. Emerg Infect Dis. 2018;24(6):1087-90. https://doi.org/10.3201/eid2406.171612.

9. Simner PJ, Miller S, Carroll KC. Understanding the promises and hurdles of metagenomic next-generation sequencing as a diagnostic tool for infectious diseases. Clin Infect Dis. 2018;66(5):778-88. https://doi.org/10.1 093/cid/cix881.

10. Hasan MR, Rawat A, Tang P, Jithesh PV, Thomas E, Tan R, et al. Depletion of human DNA in spiked clinical specimens for improvement of sensitivity of pathogen detection by next-generation sequencing. J Clin Microbiol. 2016; 54(4):919-27. https://doi.org/10.1128/JCM.03050-15.

11. Thoendel M, Jeraldo PR, Greenwood-Quaintance KE, Yao JZ, Chia N, Hanssen AD, et al. Comparison of microbial DNA enrichment tools for metagenomic whole genome sequencing. J Microbiol Methods. 2016;127: 141-5. https://doi.org/10.1016/j.mimet.2016.05.022

12. Tropini C, Earle KA, Huang KC, Sonnenburg JL. The gut microbiome: connecting spatial organization to function. Cell Host Microbe. 2017;21(4): 433-42. https://doi.org/10.1016/j.chom.2017.03.010.

13. Perez-Munoz ME, Arrieta MC, Ramer-Tait AE, Walter J. A critical assessment of the "sterile womb" and "in utero colonization" hypotheses: implications for research on the pioneer infant microbiome. Microbiome. 2017;5(1):48. https://doi.org/10.1186/s40168-017-0268-4

14. Yatera $\mathrm{K}$, Noguchi $\mathrm{S}$, Mukae $\mathrm{H}$. The microbiome in the lower respiratory tract. Respir Investig. 2018:56(6):432-9. https://doi.org/10.1016/j.resinv.2018. 08.003

15. Deng X, Achari A, Federman S, Yu G, Somasekar S, Bartolo I, et al. Metagenomic sequencing with spiked primer enrichment for viral diagnostics and genomic surveillance. Nat Microbiol. 2020;5(3):443-54. https://doi.org/10.1038/s41564-019-0637-9.

16. Marotz CA, Sanders JG, Zuniga C, Zaramela LS, Knight R, Zengler K Improving saliva shotgun metagenomics by chemical host DNA depletion. Microbiome. 2018;6(1):42. https://doi.org/10.1186/s40168-018-0426-3.

17. Nelson MT, Pope CE, Marsh RL, Wolter DJ, Weiss EJ, Hager KR, et al. Human and extracellular DNA depletion for metagenomic analysis of complex clinical infection samples yields optimized viable microbiome profiles. Cell Rep. 2019;26(8):2227-40 e2225. https://doi.org/10.1016/j.celrep.2019.01.091.

18. Gu W, Deng X, Lee M, Sucu YD, Arevalo S, Stryke D, et al. Rapid pathogen detection by metagenomic next-generation sequencing of infected body fluids. Nat Med. 2020;27(1):115-24. https://doi.org/10.1038/s41591-020-1105-z.

19. Sanderson ND, Street $T L$, Foster D, Swann J, Atkins BL, Brent AJ, et al. Realtime analysis of nanopore-based metagenomic sequencing from infected orthopaedic devices. BMC Genomics. 2018;19(1):714. https://doi.org/10.1186/ s12864-018-5094-y.

20. Loose M, Malla S, Stout M. Real-time selective sequencing using nanopore technology. Nat Methods. 2016;13(9):751-4. https://doi.org/10.1038/nmeth.3930.

21. Payne A, Holmes N, Clarke T, Munro R, Debebe BJ, Loose M. Readfish enables targeted nanopore sequencing of gigabase-sized genomes. Nat Biotechnol. 2020;39(4):442-50. https://doi.org/10.1038/s41587-020-00746-x.

22. Kovaka S, Fan Y, Ni B, Timp W, Schatz MC. Targeted nanopore sequencing by real-time mapping of raw electrical signal with UNCALLED. Nat Biotechnol. 2020;39:431-41.

23. Burnham P, Kim MS, Agbor-Enoh S, Luikart H, Valantine HA, Khush KK, et al. Single-stranded DNA library preparation uncovers the origin and diversity of ultrashort cell-free DNA in plasma. Sci Rep. 2016;6(1):27859. https://doi.org/1 0.1038 srep27859.

24. Johnson JS, Spakowicz DJ, Hong BY, Petersen LM, Demkowicz P, Chen L, et al. Evaluation of $16 S$ rRNA gene sequencing for species and strain-level microbiome analysis. Nat Commun. 2019;10(1):5029. https://doi.org/10.1038/ s41467-019-13036-1.

25. Quick J, Grubaugh ND, Pullan ST, Claro IM, Smith AD, Gangavarapu K, et al. Multiplex PCR method for MinION and Illumina sequencing of Zika and other virus genomes directly from clinical samples. Nat Protoc. 2017;12(6): 1261-76. https://doi.org/10.1038/nprot.2017.066.

26. Metsky HC, Siddle KJ, Gladden-Young A, Qu J, Yang DK, Brehio P, et al. Capturing sequence diversity in metagenomes with comprehensive and scalable probe design. Nat Biotechnol. 2019;37(2):160-8. https://doi.org/10.1 038/s41587-018-0006-X. 
27. Yang X, Li M, Liu Q, Zhang Y, Qian J, Wan X, et al. Dr.VIS v2.0: an updated database of human disease-related viral integration sites in the era of highthroughput deep sequencing. Nucleic Acids Res. 2015;43(Database issue): D887-92. https://doi.org/10.1093/nar/gku1074.

28. Horz HP, Scheer S, Huenger F, Vianna ME, Conrads G. Selective isolation of bacterial DNA from human clinical specimens. J Microbiol Methods. 2008; 72(1):98-102. https://doi.org/10.1016/..mimet.2007.10.007.

29. Li H. Minimap2: pairwise alignment for nucleotide sequences. Bioinformatics. 2018;34(18):3094-100. https://doi.org/10.1093/bioinformatics/ bty191.

30. Danecek P, Bonfield JK, Liddle J, Marshall J, Ohan V, Pollard MO, et al. Twelve years of SAMtools and BCFtools. Gigascience. 2021;10(2). https://doi. org/10.1093/gigascience/giab008.

31. Kim D, Song L, Breitwieser FP, Salzberg SL. Centrifuge: rapid and sensitive classification of metagenomic sequences. Genome Res. 2016;26(12):1721-9. https://doi.org/10.1101/gr.210641.116.

\section{Publisher's Note}

Springer Nature remains neutral with regard to jurisdictional claims in published maps and institutional affiliations.

Ready to submit your research? Choose BMC and benefit from:

- fast, convenient online submission

- thorough peer review by experienced researchers in your field

- rapid publication on acceptance

- support for research data, including large and complex data types

- gold Open Access which fosters wider collaboration and increased citations

- maximum visibility for your research: over $100 \mathrm{M}$ website views per year

At BMC, research is always in progress.

Learn more biomedcentral.com/submissions 